\title{
High-Order Nonlinear Spin-Orbit Interaction on Plasmonic Metasurfaces
}

Shumei Chen," Kingfai Li, Junhong Deng, Guixin Li, and Shuang Zhang*

KEYWORDS: plasmonic metasurface, spin-orbit interaction, second harmonic generation, optical vortex.

\begin{abstract}
In linear optics, the angular momentum of light can be easily manipulated through the optical spin-orbit interaction (SOI) in structured media such as liquid crystals, metasurfaces and forked gratings. Similarly, metasurfaces can be used to generate nonlinear optical beams with both custom-defined SAM and OAM states. However, it has been limited to a low-order process in which only a Gaussian-shape fundamental wave is used. In this work, the high-order nonlinear optical SOI effect on metasurfaces is demonstrated through the generation of multiple angular momentum states in nonlinear waves. This is achieved by exploiting the degree of freedoms provided by both the SAM and OAM states of FW and the topological charges of the plasmonic metasurfaces. The mechanism of both intrinsic and extrinsic contributions to the OAM of the nonlinear waves is revealed. High-order nonlinear SOI on metasurfaces offers new opportunities for realizing ultra-compact nonlinear vortex beams.
\end{abstract}


The spin and orbital angular momentum (SAM and OAM) of photons, which are associated with the circular polarizations and the helical wavefronts of light, ${ }^{1,2}$ have been widely studied in the fields of classical $^{3}$ and quantum optical communications, ${ }^{4-8}$ microscopy, ${ }^{9}$ micromanipulation, ${ }^{10}$ etc. The simultaneous control of both the SAM and OAM of light provides more degrees of freedom for optical information processing. ${ }^{5,11-14}$ By virtue of the optical spin-orbit interaction, ${ }^{12}$ the simultaneous control of SAM and OAM in linear optics can be easily realized by using liquid crystal based phase plates, ${ }^{12,15,16}$ photonic metasurfaces ${ }^{17-22}$ and so on. On the other hand, there have been growing interests to control $\mathrm{SAM}^{23-28}$ and $\mathrm{OAM}^{29-34}$ of light in nonlinear optical processes. For example, SAM states in harmonic generations and four-wave-mixing can be precisely controlled by optical crystals or plasmonic metasurfaces, wherein the symmetry selection rules for the nonlinear optical processes with circular polarized fundamental waves play an important role..$^{23,25,26,35}$ Using the bi-color excitation technique, the spin states of high harmonic generation (HHG) signals can be also controlled by the virtual symmetry of the electric fields of light in a gas system. ${ }^{36}$

In nonlinear optical crystals, a fundamental wave (FW) with an OAM of $l \hbar$ can excite the $n^{\text {th }}$ harmonic generations with an OAM of $n l \hbar,{ }^{37}$ which verifies the conservation law of orbital angular momentum during the nonlinear optical process. However, for the HHG process in a gas medium, the OAM states of nonlinear waves seems difficult to be predicted using the conservation law. ${ }^{38}$ Recently, the self-torque induced time-varying OAM of HHG waves in the gas was discovered. ${ }^{39}$ In addition, the OAM states of harmonic generation waves can be manipulated by introducing a topological charge of $q$ into the nonlinear medium, for example, by using the electric poling technique, which is usually utilized to generate the binary phase in the nonlinear ferroelectric crystals. ${ }^{33,34}$ However, the pixel size of the poled nonlinear crystal usually is at the micrometer scale, which puts a constraint on ultra-compact optical integrations. This constraint can be circumvented by using the newly developed nonlinear metasurfaces, with which both the phase and amplitude of the nonlinear optical waves can be controlled by 
engineering the subwavelength meta-atoms. ${ }^{40-44}$ Using nonlinear plasmonic metasurfaces consisting of split ring resonator (SRR) meta-atoms, generation of linearly polarized OAM state of nonlinear waves has been demonstrated. ${ }^{29,30}$

However, despite the extensive studies of angular momentum of light in the nonlinear optical regime, few works have been carried out on the simultaneous control of both SAM and OAM of nonlinear waves. The circularly polarized OAM states of the second harmonic generation (SHG) waves have been achieved by using the concept of nonlinear geometric phase, 31 which occurs when a FW interacts with an array of nonlinear meta-atom with certain rotational symmetries. It should be noted that the spin controlled OAM generation of the nonlinear waves on the metasurfaces has been mainly studied by using a FW with Gaussian profile. ${ }^{31}$ If the FW has both SAM and OAM states, the nonlinear optical process on the metasurface may involve a high-order spin-obit interaction process, which was only studied in linear optical regime..$^{45}$

Here, we study the high-order spin-orbit interaction (SOI) in the nonlinear optical processes on a plasmonic metasurface, in which a topological charge $q$ is encoded in an array of spatially-variant meta-atoms with three-fold rotational symmetry $(\mathrm{C} 3) .{ }^{25,27,31}$ As indicated in Figure 1 , a FW has an angular momentum state of $(\sigma, l)_{\omega}$, where $\sigma= \pm 1$ and $l$ indicate the SAM and OAM states respectively. When the FW is normally incident on the plasmonic metasurface, according to the spin controlled symmetry selection rules, ${ }^{23,25}$ the SAM of the SHG wave is expected to be flipped from $\sigma \hbar$ to $-\sigma \hbar$. Meanwhile, the OAM states of the SHG waves contain two contributions: (i) the intrinsic contribution: the SHG waves directly inherit an OAM of $2 l \hbar$ from the FW during the two photon interaction process; ${ }^{33}$ (ii) the extrinsic contribution: an additional OAM of $3 \sigma q \hbar$ arises from the phase singularity of the plasmonic metasurface. ${ }^{31}$ Therefore, generation of a SHG wave with angular momentum states of $(-\sigma, 2 l+3 \sigma q)_{2 \omega}$ is expected from the high-order nonlinear spin-orbit interaction process between the FW and the metasurface with topological charges. To the best of our knowledge, the combination of these 
two contributions in the context of metamaterials and metasurfaces have not been studied previously. It is shown that the OAM state of SHG wave can also be controlled by changing the value of $l$. As $l$ could be any integers, the OAM of the SHG wave can be tuned in a much broader range than that in ref. 31. In addition, the exact value of the OAM state of the SHG waves from the plasmonic metasurfaces cannot be measured in ref. 31 , however, this constraint can be easily solved by using the high-order nonlinear SOI process.

\section{RESULTS AND DISCUSSION}

To verify our theoretical prediction, we design the plasmonic metasurface devices with various phase singularities. In the linear optical regime, it is known that optical vortex beams with OAMs can be easily produced by using phase plates with spiral phase profiles. For a typical half-wave spiral phase plate ( $q$-plate) with topological charge $q$, when it is illuminated by circularly polarized Gaussian beam with SAM of $\sigma \hbar$, an optical vortex carrying OAM of $2 q \hbar$ with opposite SAM of $-\sigma \hbar$ can be generated. The phase of the generated vortex is then described by: $\varphi(x, y)=\exp (i 2 \sigma q \theta)$, where $\theta=\arctan (x / y)$ and $x, y$ are the Cartesian coordinates. ${ }^{12}$ The topological charge $q$ can be either positive or negative, corresponding to a clockwise or counterclockwise rotation direction of the phase change, respectively. In the nonlinear optical regime, for a $\mathrm{C} 3$ gold plasmonic meta-atom illuminated by a circularly polarized $\mathrm{FW}$, the SHG wave with the opposite handedness can be generated based on the symmetry selection rules in harmonic generations. ${ }^{23,25}$ By spatially varying the orientation of a $\mathrm{C} 3$ meta-atom with an inplane angle of $\theta$ respect to the $x$-axis, the SHG wave experiences a geometric phase term of $\exp (i 3 \sigma q \theta)$, where $\sigma$ indicates the SAM state of the FW. Hence, by continuous varying $\theta$ from 0 to $2 \pi / 3$, a complete nonlinear geometric phase ranging from 0 to $2 \pi$ will be imprinted onto the SHG waves. ${ }^{27}$

As shown in Figure 2, nine metasurfaces with topological charges $q$ ranging from $-4 / 3$ to 4/3 are designed and fabricated on ITO coated glass substrates by using the electron beam 
lithography followed by a metal lift-off process. The scanning electron microscope images of the nine metasurfaces are also shown in Figure 2. To obtain the linear optical responses of the metasurfaces, the transmission spectra are calculated and measured in both the visible and the near-infrared regimes. Two plasmonic resonances located at wavelengths around $1100 \mathrm{~nm}$ and $1400 \mathrm{~nm}$ are experimentally observed, which are due to localized plasmonic resonances at goldITO and gold-air interfaces, respectively. It can be found that the experimental results agree well with the simulated ones (details are shown in Supporting Information).

Next, we experimentally characterize their nonlinear optical properties. To do this, a circularly polarized femtosecond laser from the optical parametric system (Chameleon, Coherent) is normally incident onto the metasurfaces along the $\mathrm{C} 3$ rotational axis of the metaatom, the SHG waves with left and right circular polarizations (LCP and RCP) are recorded by using an Andor spectrometer with an EMCCD detector and imaged with a home-built imaging system (Figure 3a). For the metasurface with topological charge $q=0$, the wavelength and polarization dependent nonlinear optical responses of the SHG waves are summarized in the Supporting Information. In these measurements, it is observed that the SHG wave with opposite circular polarization to that of $\mathrm{FW}$ is much stronger than that with same polarization, agreeing with the nonlinear optical selection rules. ${ }^{23,25}$

To confirm the contributions of the topological charge $q$ of the metasurface to the nonlinear optical SOI process, the images of the SHG waves from the metasurfaces are captured in Fourier space (Figure 3b). For the metasurface with uniform phase distributions $(q=0)$, the SHG beam has a Gaussian profile. In comparison, the $q$-dependent SHG vortex beams can be observed from the metasurfaces with non-zero phase singularities. By increasing the absolute value of topological charge $|q|$ of the metasurfaces, the diameter of the observed SHG vortex beam becomes larger, indicating the increasing absolute value of the OAM. To obtain the exact values of the OAM, an on-axis interference technique based on a linearly polarized FW is utilized. The FW with horizontal $(\mathrm{H})$ polarization can be decomposed into LCP and RCP components, and 
the SHG vortex beams with OAM states of $3 q \hbar$ and $-3 q \hbar$ are generated simultaneously. Consequently, the interference between the horizontally polarized (H-) components of the two circularly polarized SHG vortex beams produces a Hermite-Gaussian (HG) pattern with a petal number of $6|q|$. From the third row of Figure 3b, i. e. for metasurfaces with topological charges of $q= \pm 1 / 3, \pm 2 / 3, \pm 1$ and $\pm 4 / 3$, the SHG waves have OAM values of $\pm \hbar, \pm 2 \hbar, \pm 3 \hbar$, and $\pm 4 \hbar$, respectively. The observed OAM states of SHG beams agree with our simulation results (shown in Figure 3c), confirming that the topological charge of the metasurface can introduce an additional OAM contribution of $|3 \sigma q \hbar|$. It should be noted that the sign of the OAM values of the SHG vortex beams cannot be distinguished by just using the on-axis interference method. These observations also agree well with results in a previous work, ${ }^{31}$ however, the sign of the topological charge $q$ of the metasurface was not considered in Reference 31 .

To unveil the physics of the high-order nonlinear optical SOI process on the plasmonic metasurfaces, a FW with OAM states is prepared when a circularly polarized Gaussian FW with a SAM of $\sigma \hbar$ passes through a vortex phase plates with topological charge $q=1$, as shown in Figure 4a. The angular momentum states of the transmitted FW becomes $(-\sigma, l)_{\omega}$ with $l=2 \sigma$. For the incident Gaussian FW with LCP and RCP states, the output angular momentum states after the vortex phase plate are $(-1,1)_{\omega}$ and $(1,-1)_{\omega}$, respectively. The SAM and OAM states of the SHG beams from the same plasmonic metasurfaces as in Figure 2 are captured and analyzed by using the on-axis interference technique. All the measured results are summarized in Figure $4 \mathrm{~b}$. For the metasurface with $q=0$, it is found that the SHG waves acquires an OAM of $2 l_{\omega} \hbar$, which obeys the conservation law of OAMs in the SHG process. The SHG beams generated by the vortex FW through metasurfaces with topological charges $q= \pm 1 / 3, \pm 2 / 3, \pm 1$ and $\pm 4 / 3$ show great contrast to that with a Gaussian FW shown in Figure 3b. For example, under the excitation of FW with angular momentum state of $(1,-1)_{\omega}$, the metasurface with topological charge $q=2 / 3$ produces a Gaussian profile SHG wave. Such a Gaussian SHG signal can be observed as well when the angular momentum state of FW is flipped to $(-1,1)_{\omega}$. To 
determine the exact OAMs values, we also perform the on-axis interference measurement by using horizontal polarized FW. In this case, after passing through the vortex phase plates, the output FW can be regarded as the superposition of two states $(-1,1)_{\omega}$ and $(1,-1)_{\omega}$. The interference pattern of the SHG vortices obtained from the two states are shown in the third row in Figure 4b. When the topological charges of the metasurface are increased from $-4 / 3$ to $4 / 3$, the petal number of the on-axis interference patterns are decreased from 12 to zero and then increased to 4. Therefore, the measured absolute OAM values of the SHG waves should be $6 \hbar$, $5 \hbar, 4 \hbar, 3 \hbar, 2 \hbar, \hbar, 0, \hbar, 2 \hbar$, respectively. As shown in Figure $4 \mathrm{c}$, these results can be nicely explained by the theoretical prediction of the SAM and OAM states based on the formula ( $-\sigma$, $2 l+3 \sigma q)_{2 \omega}$. Specifically, for FW at angular momentum state of $(1,-1)_{\omega}$, the plasmonic metasurfaces with increasing topological charges $q=-4 / 3$ to $4 / 3$ produce SHG waves with OAM of $-6 \hbar,-5 \hbar,-4 \hbar,-3 \hbar,-2 \hbar,-\hbar, 0, \hbar, 2 \hbar$, respectively. In comparison, for FW with angular momentum state of $(1,-1)_{\omega}$, the predicted OAM values of the SHG waves are of $6 \hbar, 5 \hbar, 4 \hbar, 3 \hbar$, $2 \hbar, \hbar, 0,-\hbar,-2 \hbar$, respectively. The formula $(-\sigma, 2 l+3 \sigma q) 2 \omega$ is also confirmed by using FW with angular momentum state of $(-1,2)_{\omega}$ and $(1,-2)_{\omega}$, where the details can be found in Supporting Information.

To better understand the nonlinear SOI processes, we theoretically analyzed the field distribution of the SHG waves from the nine metasurfaces by using FW with different angular momentum states (Figure $3 \mathrm{c}$ and Figure $4 \mathrm{c}$ ). For FWs with OAM and SAM states $(\sigma, l)_{\omega}$, the SHG vortices from each C3 metasurface are assumed to have a Laguerre-Gaussian (LG) beam profile with SAM state $-\sigma$ and azimuthal mode number $|2 l+3 \sigma q|$. Then, the intensity distribution of the SHG waves can be described by following equation:

$$
I_{S H G} \propto\left|E_{S H G}(r, \theta)\right|^{2} \propto\left|r^{|2 l+3 \sigma q|} \cdot e^{i(2 l+3 \sigma q) \theta} \cdot e^{-r^{2} / w^{2}}\right|^{2}
$$

where $r$ is the transverse coordinate, $w$ is the beam waist of the LG beam, and $\theta=\arctan (x / y)$ is the phase angle. Therefore, by substituting the SAM and OAM states $(\sigma, l)_{\omega}$ into Equation 1 , 
we can obtain the intensity distribution of the SHG waves $I_{S H G}$ for a circularly polarized FW. In the meanwhile, the on-axis interference pattern can be calculated by decomposing linear polarized FW into the two CP states and then adding the electric field of SHG waves of the two components together, which leads to the typical Hermite-Gaussian (HG) beam modes as shown in the third row in Figure 3c and Figure 4c. Detailed steps of the calculation can be found in Supporting Information.

\section{CONCLUSION}

In summary, we have experimentally studied the high-order nonlinear optical spin-orbit interaction in the SHG process on plasmonic metasurfaces consisting of gold meta-atoms with three-fold rotational symmetry. Using the concept of nonlinear geometric phase, the SHG beams with spin-locked OAM state can be generated on the gold metasurface under the excitation of the fundamental waves with both spin and orbital angular momentum. A generalized principle is proposed to predict the total angular momentum states of the SHG waves. The proposed metasurface platform can simultaneously generate and measure the angular momentum states of the SHG waves. By integrating the plasmonic metasurface with conventional nonlinear crystals, such as Lithium niobate, the efficiency of the SHG vortex can be further increased. Our findings will not only benefit the understanding of physics of highorder nonlinear spin-orbit interaction of light, but also open new avenues for designing highdimensional nonlinear optical sources for information processing in either classical or quantum regimes. 


\section{METHODS}

Fabrication of the Plasmonic Metasurfaces. The gold plasmonic metasurfaces are fabricated through the electron-beam lithography (EBL) and a gold lift-off process: first, a thin resist (PMMA) layer with thickness of $120 \mathrm{~nm}$ is spin-coated onto the ITO glass substrate; The metasurface with different topological charges are patterned onto the resist layer by the EBL process; A gold layer with thickness of $30 \mathrm{~nm}$ is then deposited onto the resist patterns through electron-beam evaporation and the resist layer is finally removed by using acetone solutions. The length and width of the arms of the $\mathrm{C} 3$ meta-atoms are $160 \mathrm{~nm}$ and $80 \mathrm{~nm}$, respectively. The center to center distance of the $\mathrm{C} 3$ meta-atoms is $500 \mathrm{~nm}$.

Nonlinear Optical Experiments. The near-infrared femtosecond laser has a repetition frequency of $80 \mathrm{MHz}$ and a pulse width $\sim 250 \mathrm{fs}$. As shown in Figure 3a, the SAM state of the FW is controlled by a linear polarizer and a quarter wave plate (QWP), then the FW at wavelength of $1064 \mathrm{~nm}$ is focused onto the metasurface by a lens with focal length of $50 \mathrm{~mm}$ and numerical aperture N.A. $=0.05$. After passing through the metasurfaces, the SHG waves are collected by an objective lens $($ N.A. $=0.25)$ and the polarization state of the SHG waves are analyzed by the second quarter-wave plate and a linear polarizer. A color filter (Thorlabs FESH800) is used to block the fundamental wave at NIR wavelength range. Finally, the SHG waves are recorded by a sCMOS camera. To control the OAM states of the FW, an additional $q$-plate with topological charge $m$ is inserted after the first quarter wave plate, as indicated in Figure 4a. As the vortex plate (VP) at hand is designed at fundamental wavelength of $1064 \mathrm{~nm}$, which doesn't overlap with the plasmonic resonances of the metasurfaces, so the SHG efficiency can be greatly improved by switching the fundamental wavelength from $1064 \mathrm{~nm}$ to $1225 \mathrm{~nm}$ (see Supporting Information S3). 


\section{Figures and Captions}

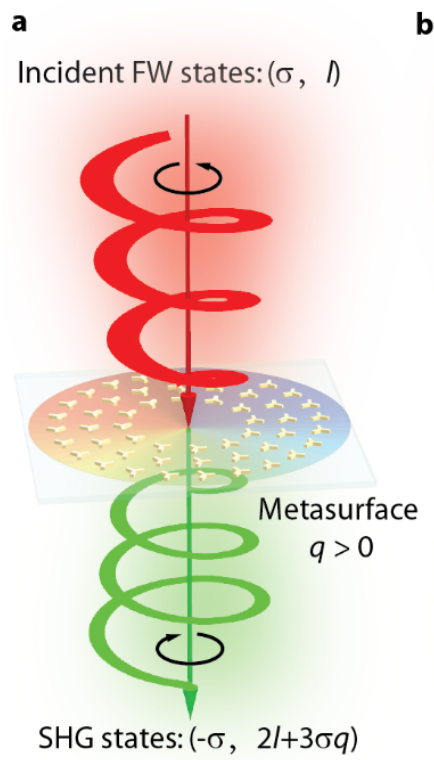

b

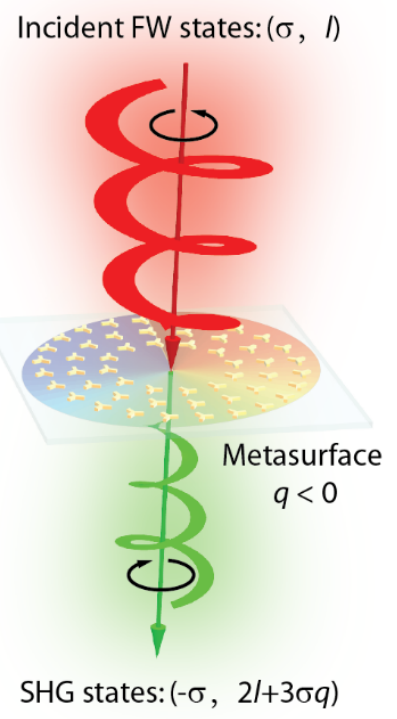

Figure 1. High-order nonlinear optical spin-orbit interaction on the plasmonic metasurfaces. (a) and (b) The plasmonic metasurfaces, consisting of gold meta-atoms with three-fold rotational symmetry, have an either positive or negative topological charge $q$. For fundamental wave $(\mathrm{FW})$ with spin and orbital angular momentum $(\sigma, l)_{\omega}$, the second harmonic generation (SHG) waves have opposite SAM to that of FW due to the symmetry selection rules. In addition, the SHG wave acquires OAM from the contributions from both the fundamental wave $(2 l \hbar)$ and the metasurfaces which have spin controlled nonlinear geometric phase singularities $(3 \sigma q \hbar)$. The direction of the black arrows indicate the SAM states of the FW (frequency: $\omega$ ) and SHG wave (frequency: $2 \omega$ ). The handedness and size of the spirals indicates the OAM properties of the light. 

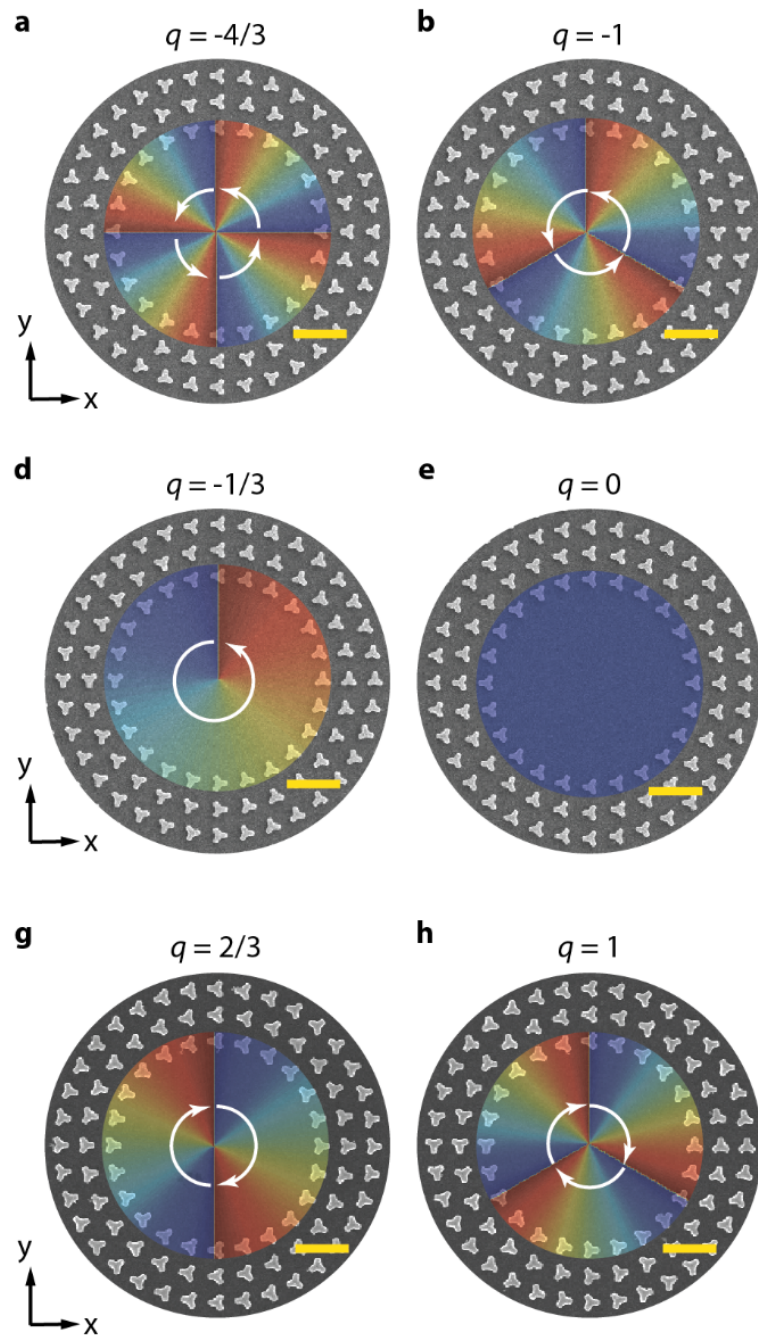

h

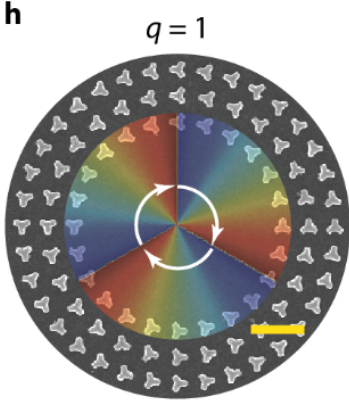

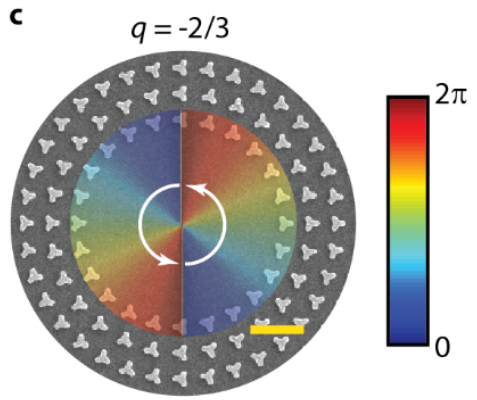

$\mathbf{f}$
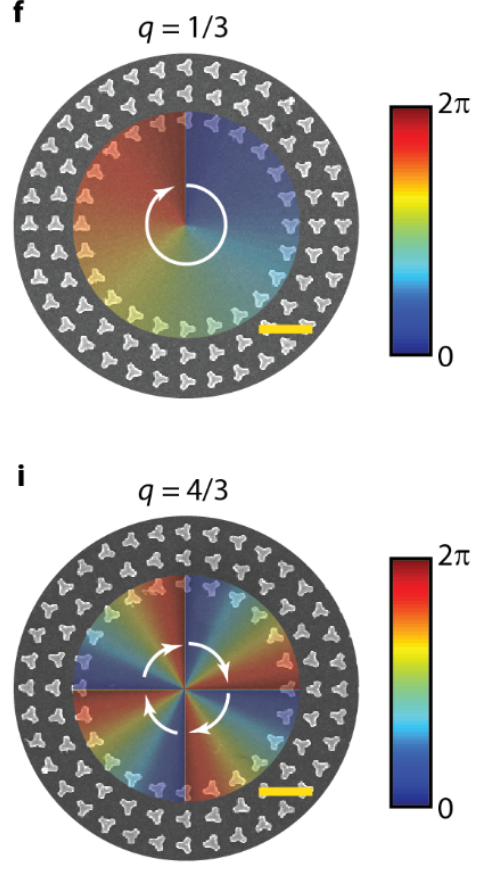

Figure 2. Plasmonic metasurfaces with different topological charges. (a-i) Scanning electron microscopy images of the metasurfaces, which consist of gold meta-atoms with three-fold rotational symmetry. The topological charges of the metasurfaces are from $q=-4 / 3$ to $4 / 3$. The insert color maps indicate the nonlinear geometric phase distribution produced in the SHG processes. (a-d) For $q<0$, the phase changes from 0 to $2 \pi$ in the counterclockwise direction; (e) Metasurface with uniform phase distribution $(q=0)$; ( $\mathrm{f}-\mathrm{i})$ For $q>0$, the nonlinear geometric phase varies from 0 to $2 \pi$ in the clockwise direction, as indicated by the white arrows. Scale bar: $1 \mu \mathrm{m}$. 
a

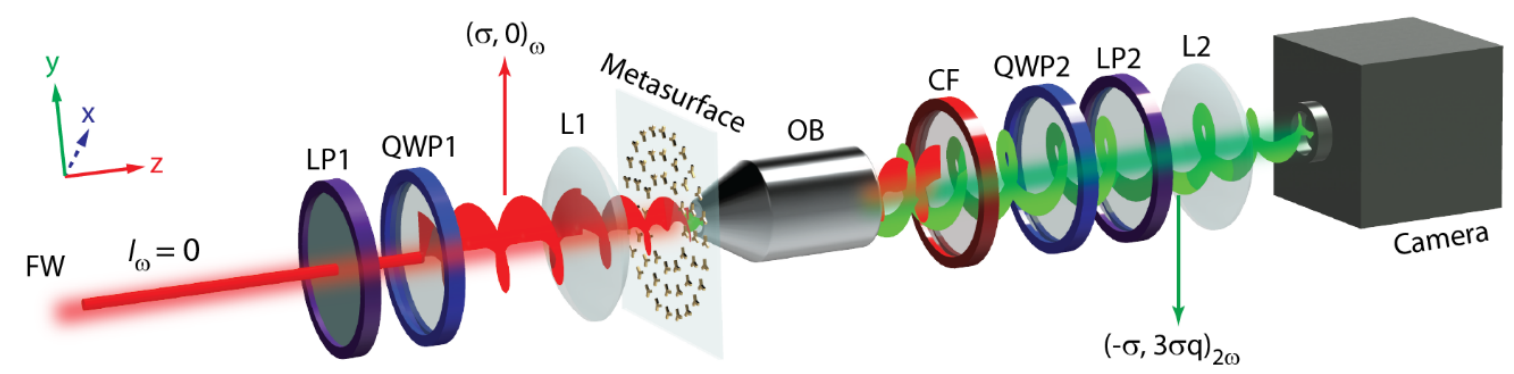

b
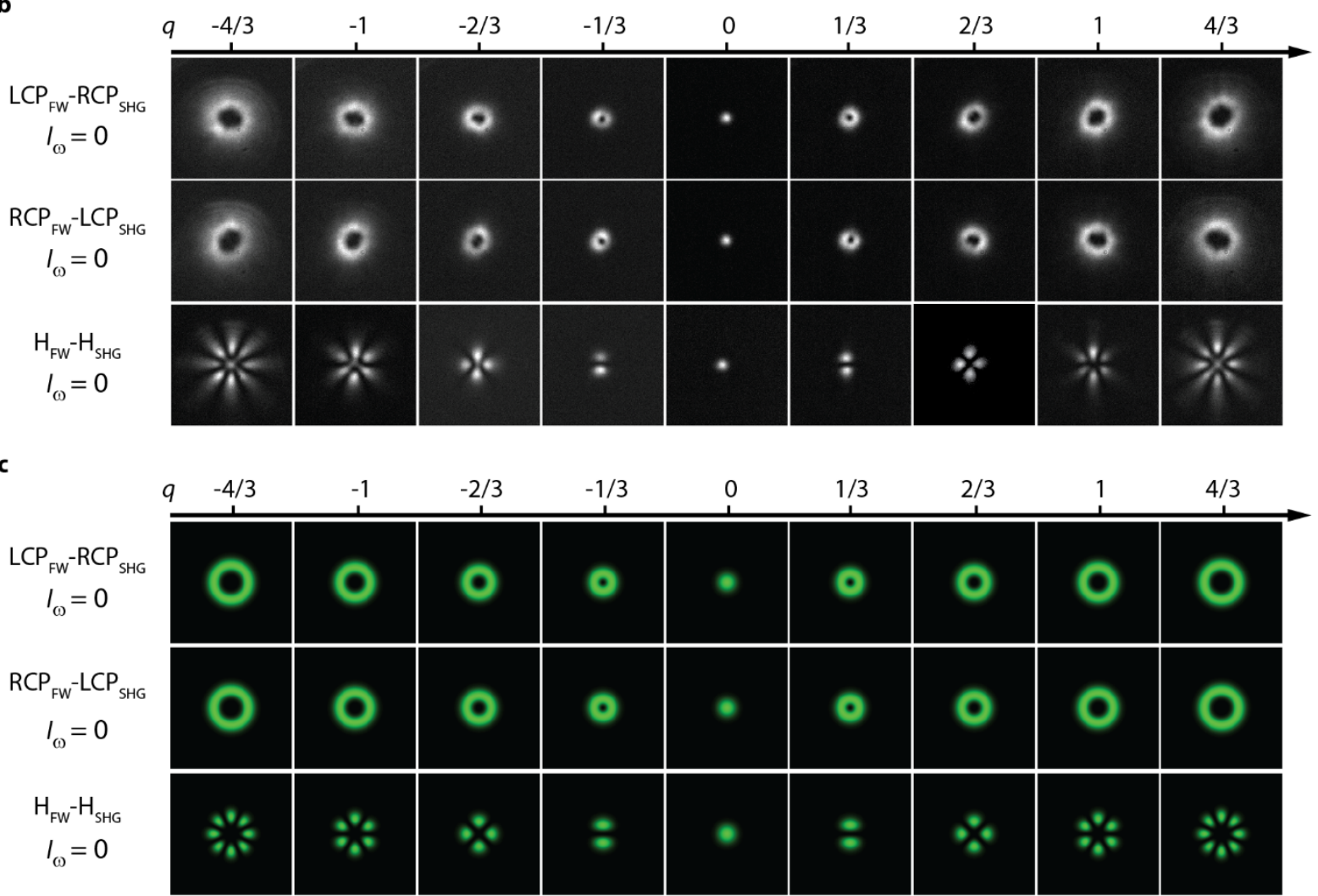

Figure 3. Nonlinear optical spin-orbit interaction through the SHG process on the plasmonic metasurfaces. (a) Experimental setup for generation and measurements of the spin controlled angular momentum states of the SHG waves. LP1 and LP2: linearly polarizers; QWP1 and QWP2: quarter wave plates; L1 and L2: lens; OB: objective lens. (b) Under the excitation of circularly polarized Gaussian FW, the images of the SHG vortex beams with both SAM and OAM are captured by using a camera. (c) Simulated beam profiles of the SHG waves with different angular momentum states. The first and second rows in (b) and (c) correspond to the FW with an angular momentum state of $(\sigma, 0)_{\omega}$. For the third row, both the incident FW and SHG waves are horizontally polarized. The SHG waves with opposite OAM values have an onaxis interference, from which the OAM value can be retrieved. 
a

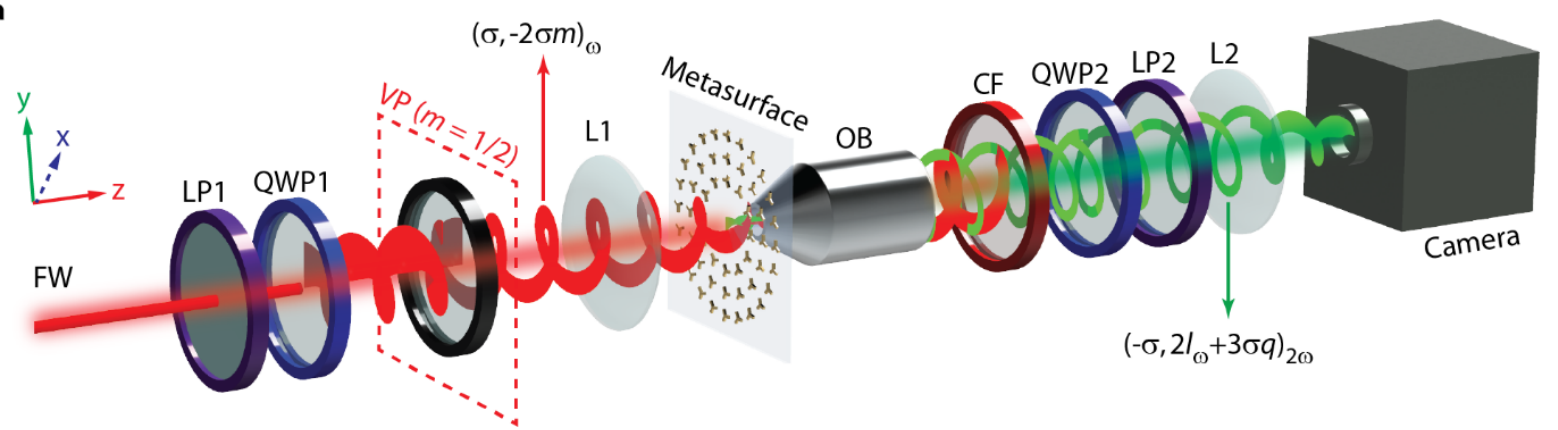

b
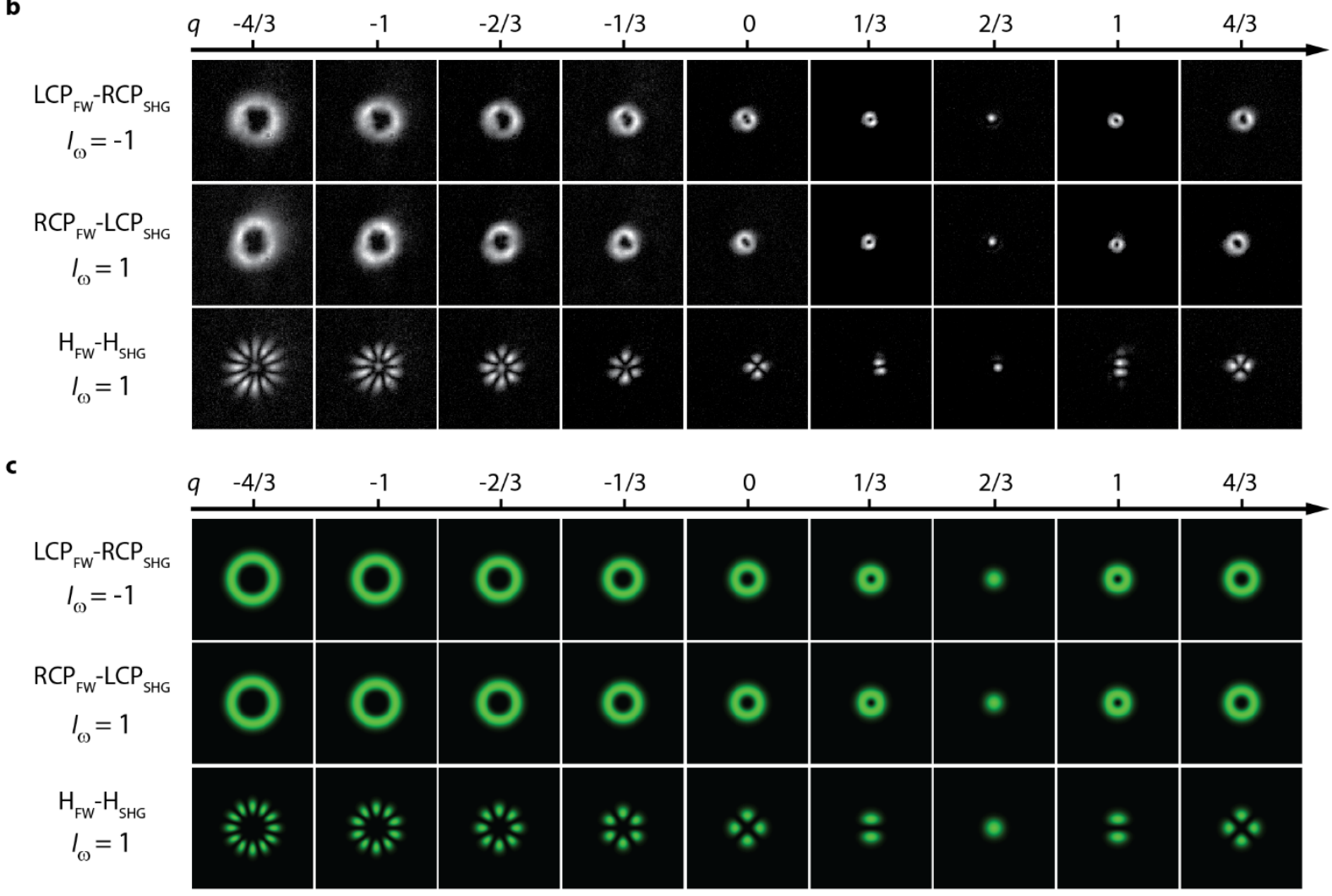

Figure 4. High-order nonlinear optical spin-orbit interaction through the SHG process on the plasmonic metasurfaces. (a) Experimental setup for generation and measurement of the SHG waves. The OAM state of FW is generated it passes through the vortex plate (VP). LP1 and LP2: linearly polarizers; QWP1 and QWP2: quarter wave plates; L1 and L2: lens; OB: objective lens. (b) Under the excitation of FW with both spin and orbital angular momentum, the images of the SHG vortex beams with various angular momentum states are recorded. (c) Simulated beam profile of the SHG waves with different angular momentum states. The first and second rows in (b) and (c) correspond to the FW with an angular momentum state of $(\sigma, l)_{\omega}$. For the third row, both the incident FW and SHG waves are horizontally polarized. The SHG waves have angular momentum state of $(-\sigma, 2 l+3 \sigma q)_{\omega}$, which can be experimentally verified using the on-axis interference technique. 


\section{ASSOCIATED CONTENT}

\section{Supporting Information}

The supporting information is available free of charge via the internet at http://pubs.acs.org. Linear optical properties of the metasurfaces; Characterization of the SHG waves from the plasmonic metasurfaces; The wavelength dependent nonlinear optical responses of the metasurfaces; High-order nonlinear optical SOI process with angular momentum states of the FW $(1,-2)_{\omega}$ and $(-1,2)_{\omega}$; Numerical simulation of the SHG waves.

\section{AUTHOR INFORMATION}

\section{Corresponding Authors}

\section{Shumei Chen}

School of Science, Harbin Institute of Technology, Shenzhen, 518055, China

Key Laboratory of Micro-Nano Optoelectronic Information System of Ministry of Industry and Information Technology, Harbin Institute of Technology, Shenzhen, 518055, China

E-mail: chenshumei@hit.edu.cn

\section{Shuang Zhang}

School of Physics \& Astronomy, University of Birmingham, Birmingham, B15 2TT, UK

E-mail: s.zhang@bham.ac.uk

\section{Authors}

Kingfai Li - Department of Materials Science and Engineering, Southern University of Science and Technology, Shenzhen, 518055, China.

Junhong Deng - Department of Materials Science and Engineering, Shenzhen Institute for Quantum Science and Engineering, Southern University of Science and Technology, Shenzhen, 518055, China.

Guixin Li - Department of Materials Science and Engineering, Shenzhen Institute for Quantum Science and Engineering, Southern University of Science and Technology, Shenzhen 518055, China. 


\section{Author Contributions}

S. C. and K. L. contributed equally. S. C. conceived the idea and carried out the linear and nonlinear simulations. J. D. fabricated the metasurfaces. K. L. and S. C. measured the linear and nonlinear optical properties of the metasurfaces. S. C. and S. Z. supervised the project. All authors contributed to the data analysis and manuscript writing.

\section{Notes}

The authors declare no competing financial interests.

\section{ACKNOWLEDGMENTS}

S. C. acknowledges the financial support from Shenzhen Peacock Program. S. Z. acknowledges the funding from the European Research Council (ERC) under the European Union's Horizon 2020 research and innovation programme (grant agreement No. 648783). G. L. is financially supported by National Natural Science Foundation of China (91950114 and 11774145), Guangdong Provincial Innovation and Entrepreneurship Project (2017ZT07C071).

\section{REFERENCES}

1. Poynting, J. H. The wave motion of a revolving shaft, and a suggestion as to the angular momentum in a beam of circularly polarised light. Proc. R. Soc. London Ser. A 1909, 82, 560 .

2. Allen, L.; Beijersbergen, M. W.; Spreeuw, R.; Woerdman, J. Orbital angular momentum of light and the transformation of Laguerre-Gaussian laser modes. Phys. Rev. A 1992, 45, 8185 .

3. Wang, J.; Yang, J. Y.; Fazal, I. M.; Ahmed, N.; Yan, Y.; Huang, H.; Ren, Y. X.; Yue, Y.; Dolinar, S.; Tur, M.; Willner, A. E. Terabit free-space data transmission employing orbital angular momentum multiplexing. Nat. Photon. 2012, 6, 488.

4. Wang, X. L.; Cai, X. D.; Su, Z. E.; Chen, M. C.; Wu, D.; Li, L.; Liu, N. L.; Lu, C. Y.; Pan, J. W. Quantum teleportation of multiple degrees of freedom of a single photon. Nature 2015, $518,516$.

5. Vallone, G.; D’Ambrosio, V.; Sponselli, A.; Slussarenko, S.; Marrucci, L.; Sciarrino, F.; Villoresi, P. Free-space quantum key distribution by rotation-invariant twisted photons. Phys. Rev. Lett. 2014, 113, 060503. 
6. Nagali, E.; Sansoni, L.; Sciarrino, F.; Martini, F. D.; Marrucci, L.; Piccirillo, B.; Karimi, E.; Santamato, E. Optimal quantum cloning of orbital angular momentum photon qubits through Hong-Ou-Mandel coalescence. Nat. Photon. 2009, 3, 720.

7. Leach, J.; Jack, B.; Romero, J.; Jha, A. K.; Yao, A. M.; Arnold, S. F.; Ireland, D. G.; Boyd, R. W.; Barnett, S. M.; Padgett, M. J. Quantum correlations in optical angle-orbital angular momentum variables. Science 2010, 329, 662.

8. Mair, A.; Vaziri, A.; Weihs, G.; Zeilinger, A. Entanglement of the orbital angular momentum states of photons. Nature 2001, 412, 313.

9. Schneider, J.; Zahn, J.; Maglione, M.; Sigrist, S. J.; Marquard, J.; Chojnacki, J.; Kräusslich, H. G.; Sahl, S. J.; Engelhardt, J.; Hell, S. W. Ultrafast, temporally stochastic STED nanoscopy of millisecond dynamics. Nat. Methods 2015, 12, 827.

10. Padgett, M. J.; Bowman, R. Tweezers with a twist. Nat. Photon. 2011, 5, 343.

11. Simpson, N. B.; Dholakia, K.; Allen, L.; Padgett, M. J. Mechanical equivalence of spin and orbital angular momentum of light: an optical spanner. Opt. Lett. 1997, 22, 52.

12. Marrucci, L.; Manzo, C.; Paparo, D. Optical spin-to-orbital angular momentum conversion in inhomogeneous anisotropic media. Phys. Rev. Lett. 2006, 96, 163905.

13. Zhan, Q. W. Cylindrical vector beams: from mathematical concepts to applications. Adv. Opt. Photon. 2009, $1,1$.

14. Yao, A. M.; Padgett, M. J. Orbital angular momentum: origins, behavior and applications. Adv. Opt. Photon. 2011, 3, 161.

15. Leach, J.; Gibson, G. M.; Padgett, M. J. Generation of achromatic Bessel beams using a compensated spatial light modulator. Opt. Express 2006, 14, 5581.

16. Davis, J. A.; Cottrell, D. M.; Zinn, J. M. Direct generation of abruptly focusing vortex beams using a 3/2 radial phase-only pattern. Appl. Opt. 2013, 52, 1888.

17. Yu, N. F.; Genevet, P.; Kats, M. A.; Aieta, F.; Tetienne, J. P.; Capasso, F.; Gaburro, Z. Light propagation with phase discontinuities: generalized laws of reflection and refraction. Science 2011, 334, 333.

18. Li, G. X.; Kang, M.; Chen, S. M.; Zhang, S.; Pun, E. Y. B.; Cheah, K. W.; Li, J. Spinenabled plasmonic metasurfaces for manipulating orbital angular momentum of light. Nano Lett. 2013, 13, 4148.

19. Chen, S. M.; Cai, Y.; Li, G. X.; Zhang, S.; Cheah, K. W. Geometric metasurface fork gratings for vortex-beam generation and manipulation. Laser \& Photon. Rev. 2016, 10, 322.

20. Maguid, E.; Yulevich, I.; Veksler, D.; Kleiner, V.; Brongersma, M. L.; Hasman, E. Photonic spin-controlled multifunctional shared-aperture antenna array. Science 2016, 352, 1202.

21. Devlin, R. C.; Ambrosio, A.; Rubin, N. A.; Mueller, J. B.; Capasso, F. Arbitrary spin-toorbital angular momentum conversion of light. Science 2017, 358, 896.

22. Karimi, E.; Schulz, S. A.; De Leon, I.; Qassim, H.; Upham, J.; Boyd, R. W. Generating optical orbital angular momentum at visible wavelengths using a plasmonic metasurface. Light Sci. Appl. 2014, 3, e167.

23. Armstrong, J. A.; Bloembergen, N.; Ducuing, J.; Pershan, P. S. Interactions between light waves in a nonlinear dielectric. Phys. Rev. 1962, 127, 1918. 
24. Fleischer, A.; Kfir, O.; Diskin, T.; Sidorenko, P.; Cohen, O. Spin angular momentum and tunable polarization in high-harmonic generation. Nat. Photon. 2014, 8, 543.

25. Chen, S. M.; Li, G. X.; Zeuner, F.; Wong, W. H.; Pun, E. Y. B.; Zentgraf, T.; Cheah, K. W.; Zhang, S. Symmetry-selective third-harmonic generation from plasmonic metacrystals. Phys. Rev. Lett. 2014, 113, 033901.

26. Konishi, K.; Higuchi, T.; Li, J.; Larsson, J.; Ishii, S.; Gonokami, M. K. Polarizationcontrolled circular second-harmonic generation from metal hole arrays with threefold rotational symmetry. Phys. Rev. Lett. 2014, 112, 135502.

27. Li, G. X.; Chen, S. M.; Pholchai, N.; Reineke, B.; Wong, W. H.; Pun, E. Y. B.; Cheah, K. W.; Zentgraf, T.; Zhang, S. Continuous control of the nonlinearity phase for harmonic generations. Nat. Mater. 2015, 14, 607.

28. Ye, W. M.; Zeuner, F.; Li, X.; Reineke, B.; He, S.; Qiu, C. W.; Liu, J.; Wang, Y.T.; Zhang, S.; Zentgraf, T. Spin and wavelength multiplexed nonlinear metasurface holography. Nat. Commun. 2016, 7, 11930.

29. Segal, N.; Keren-Zur, S.; Hendler, N.; Ellenbogen, T. Controlling light with metamaterialbased nonlinear photonic crystals. Nat. Photon. 2015, 9, 180.

30. Keren-Zur, S.; Avayu, O.; Michaeli, L.; Ellenbogen, T. Nonlinear beam shaping with plasmonic metasurfaces. ACS Photon. 2016, 3, 117.

31. Li, G. X.; Wu, L.; Li, K. F.; Chen, S. M.; Schlickriede, C.; Xu, Z. J.; Huang, S. Y.; Li, W. D.; Liu, Y. J.; Pun, E. Y. B.; Zentgraf, T.; Cheah, K. W.; Luo, Y.; Zhang, S. Nonlinear metasurface for simultaneous control of spin and orbital angular momentum in second harmonic generation. Nano Lett. 2017, 17, 7974.

32. Li, G. X.; Chen, S. M.; Cai, Y.; Zhang, S.; Cheah, K. W. Third harmonic generation of optical vortices using holography-based gold-fork microstructure. Adv. Opt. Mater. 2014, 2,389 .

33. Bloch, N. V.; Shemer, K.; Shapira, A.; Shiloh, R.; Juwiler, I.; Arie, A. Twisting light by nonlinear photonic crystals. Phys. Rev. Lett. 2012, 108, 233902.

34. Wei, D. Z.; Wang, C. W.; Xu, X. Y.; Wang, H. J.; Hu, Y. L.; Chen, P. C.; Li, J. W.; Zhu, Y.Z.; Xin, C.; Hu, X. P.; Zhang, Y.; Wu, D.; Chu, J. R.; Zhu, S. N.; Xiao, M. Efficient nonlinear beam shaping in three-dimensional lithium niobate nonlinear photonic crystals. Nat. Commun. 2019, 10, 4193.

35. Li, G. X.; Sartorello, G.; Chen, S. M.; Nicholls, L. H.; Li, K. F.; Zentgraf, T.; Zhang, S.; Zayats, A. V. Spin and geometric phase control four-wave mixing from metasurfaces. Laser \& Photon. Rev. 2018, 12, 1800034.

36. Dorney, K. M.; Rego, L.; Brooks, N. J.; San Román, J.; Liao, C. T.; Ellis, J. L.; Zusin, D.; Gentry, C.; Nguyen, Q. L.; Shaw, J. M.; Picón, A.; Plaja, L.; Kapteyn, H. C.; Murnane, M. M.; Hernández-García, C. Controlling the polarization and vortex charge of attosecond high-harmonic beams via simultaneous spin-orbit momentum conservation. Nat. Photon. 2019, 13, 123.

37. Courtial, J.; Dholakia, K.; Allen, L.; Padgett, M. J. Second-harmonic generation and the conservation of orbital angular momentum with high-order Laguerre-Gaussian modes. Phys. Rev. A 1997, 56, 4193.

38. Zürch, M.; Kern, C.; Hansinger, P.; Dreischuh, A.; Spielmann, C. Strong-field physics with singular light beams. Nat. Phys. 2012, 8, 743. 
39. Rego, L.; Dorney, K. M.; Brooks, N. J.; Nguyen, Q. L.; Liao, C. T.; San Román, J.; Couch, D. E.; Liu, A.; Pisanty, E.; Lewenstein, M.; Plaja, L.; Kapteyn, H. C.; Murnane, M. M.; Hernández-García, C. Generation of extreme-ultraviolet beams with time-varying orbital angular momentum. Science 2019, 364, eeaw9486.

40. Kauranen, M.; Zayats, A. V. Nonlinear plasmonics. Nat. Photon. 2012, 6, 737.

41. Lapine, M.; Shadrivov, I. V.; Kivshar, Y. S. Colloquium: nonlinear metamaterials. Rev. Mod. Phys. 2014, 86, 1093.

42. Li, G. X.; Zhang, S.; Zentgraf, T. Nonlinear photonic metasurfaces. Nat. Rev. Mater. 2017, 2,17010 .

43. Liu, L.; Wu, L.; Zhang, J.; Li, Z.; Zhang, B.; Luo, Y. Backward phase matching for second harmonic generation in negative-index conformal surface plasmonic metamaterials. $A d v$. Sci. 2018, 5, 1800661.

44. Hu, H.; Zhang, J.; Maier, S. A.; Luo, Y. Enhancing third-harmonic generation with spatial nonlocality. ACS Photon. 2018, 5, 592.

45. Milione, G.; Evans, S.; Nolan, D. A.; Alfano, R. R. Higher Order Pancharatnam-Berry Phase and the Angular Momentum of Light. Phys. Rev. Lett. 2012, 108, 190401. 
High-Order Nonlinear Spin-Orbit Interaction on Plasmonic Metasurfaces

Shumei Chen, ${ }^{*}$ Kingfai Li, Junhong Deng, Guixin Li, and Shuang Zhang*

\section{ToC figure}
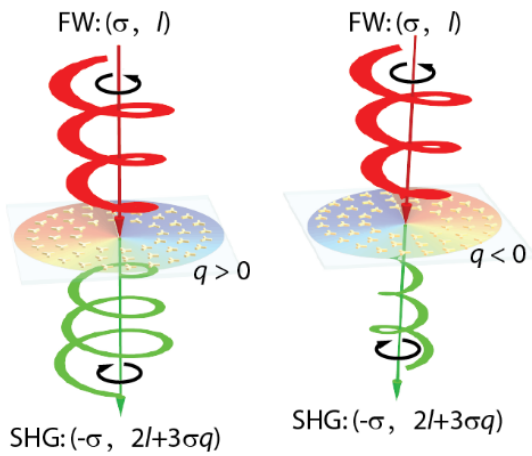